\title{
Distúrbio mineral e ósseo após o transplante renal
}

Guidelines for bone and mineral disorders after kidney transplantation

\section{Autores: \\ Ana Paula Santana \\ Gueiros \\ Carolina Lara Neves \\ Elisa de Albuquerque \\ Sampaio \\ Melani Ribeiro \\ Custódio}

\section{No $1^{\circ}$ ano de Transplante Renal}

1 Monitorar os níveis séricos de cálcio (Ca) e fósforo (P) uma vez por semana até a estabilização (Evidência). Em seguida, realizar dosagens mensais até o final do $1^{\circ}$ ano (Opinião).

2 Monitorar os níveis séricos de fosfatase alcalina (FA) e paratormônio (PTH) no momento do transplante renal (TxR) e com 6 e 12 meses (Opinião).

3 O tratamento das anormalidades do $\mathrm{Ca}, \mathrm{P}$ e PTH segue as diretrizes para DRC I-V (Evidência).

a. A paratireoidectomia (PTx) está indicada quando houver hipercalcemia grave, em qualquer momento após o TxR, ou persistência do hiperparatireoidismo secundário (HPS), preferencialmente após 1 ano do TxR (Evidência).

4 Dosar 25-hidroxivitamina D (25-vit D) no início do TxR e a cada 6 meses. Repor a hipovitaminose D conforme as recomendações para pacientes com DRC I-V, monitorando o nível sérico de 3/3 meses (Opinião).

5 Utilizar a menor dose possível de corticosteroides (Evidência).

6 A densitometria óssea (DEXA) deve ser realizada até o $3^{\circ}$ mês após o TxR em pacientes com DRC I-III T, é repetida ao final do $1^{\circ}$ ano independente de qualquer intervenção (Opinião).

7 Na presença de perda de massa óssea evidenciada pela DEXA em pacientes com DRC I-III T, considerar o uso de vitamina $\mathrm{D}_{2}$ ou $\mathrm{D}_{3}$, calcitriol ou bisfosfonatos (Opinião).
8 Considerar a possibilidade de realizar a biópsia óssea antes do uso de bisfosfonatos para afastar a presença de baixa remodelação óssea ou defeito de mineralização (Opinião).

\section{Após o $1^{\circ}$ ano de Transplante Renal}

1 Monitorar os níveis séricos de $\mathrm{Ca}, \mathrm{P}$, FA e PTH de acordo com o estágio da DRC. A frequência das dosagens deverá ser aumentada na vigência de intervenção terapêutica.

a. DRC I-III T: Ca, P e FA 6-12 meses PTH 1x/ano

b. DRC IV T: Ca , P e FA 3-6 meses PTH 3-6 meses

c. DRC V T: Ca, P e FA 1-3 meses PTH 3/3 meses

2 O tratamento das anormalidades do $\mathrm{Ca}, \mathrm{P}, \mathrm{FA}$ e PTH segue as diretrizes para DRC I-V (Evidência) e a orientação de PTx mantém-se conforme o $1^{\circ}$ ano de TxR (Evidência).

3 Monitorar os níveis de 25-vit D uma vez por ano. Nos pacientes que realizaram reposição de vitamina $\mathrm{D}_{2}$ ou $\mathrm{D}_{3}$, o controle deve ser feito de $6 / 6$ meses, e para os paciente em reposição e/ou manutenção deve ser de $3 / 3$ meses (Opinião).

4 Em pacientes DRC I-III T, a DXA deve ser realizada a cada 2 anos, se houver perda de massa óssea no $1^{\circ}$ ano do TxR, e anualmente nos pacientes que estiverem em tratamento da osteodistrofia renal (Opinião).

5 No tratamento da perda de massa óssea em pacientes com DRC I-III T 
pode-se considerar o uso de vitamina $\mathrm{D}_{2}$ ou $\mathrm{D}_{3}$, calcitriol ou bisfofonatos (Opinião). Em pacientes com DRC IV-V T, o tratamento deve seguir as mesmas recomendações para DRC IV-V.

6 A indicação de biópsia óssea segue as mesmas recomendações do $1^{\circ}$ ano do TxR.

\section{RACIONAL}

O transplante renal (TxR) é a melhor alternativa de tratamento para os pacientes com DRC avançada. Entretanto, apesar do sucesso dessa terapia, os pacientes submetidos ao TxR podem apresentar elevada incidência de complicações, dentre elas a persistência da doença óssea, piorando a qualidade de vida e aumentando a morbimortalidade. O TxR bem sucedido geralmente corrige ou melhora os distúrbios do metabolismo mineral e ósseo (DMO) da DRC, e a persistência dessas alterações são determinadas pela magnitude das anormalidades no período dialítico, disfunção do enxerto e ação de drogas imunossupressoras.

Muitas das alterações do metabolismo mineral que ocorrem logo após o TxR tendem a se normalizar no decorrer do primeiro ano. A hipofosfatemia, acompanhada de fosfatúria, ocorre em $90 \%$ dos pacientes transplantados, e geralmente apresenta resolução do quadro até o terceiro mês, permanecendo no limite inferior da normalidade. ${ }^{1}$ Entretanto, alguns pacientes, mesmo com níveis séricos normais de $\mathrm{P}$, continuam apresentando fosfatúria significativa, causada por concentrações séricas elevadas de PTH e do fator de crescimento de fibroblastos (FGF-23), ${ }^{2,3}$ que geralmente se normalizam no final do primeiro ano. A monitoração da hipofosfatemia prolongada é importante principalmente pelo risco que o paciente apresenta de evoluir para um defeito de mineralização óssea, diagnóstico que deve ser confirmado por meio da biópsia óssea. O tratamento da hipofosfatemia é questionado, limitando-se a casos graves e com pequenas doses de reposição, visto que esta terapêutica provavelmente aumentará a secreção de FGF-23, prolongando esse processo.

Os níveis séricos de Ca geralmente se elevam após 2 meses do TxR, ficando na faixa superior da normalidade. Entretanto, alguns pacientes persistem com hipercalcemia, mesmo após o término do primeiro ano, frequentemente associada com a persistência do HPS. Deve-se, também, afastar outras causas de hipercalcemia, como a presença de neoplasias, reabsorção de calcificações ectópicas e acidose metabólica.
A hipercalcemia crônica é fator de risco de mortalida$\mathrm{de}^{4}$ e favorece o desenvolvimento de nefrocalcinose, com menor sobrevida do enxerto..$^{5-7}$

A FA e a óssea também tendem a se normalizar após o primeiro ano do TxR, exceto quando há a persistência do HPS. Com a restauração da função renal, os níveis de PTH caem rapidamente, em torno de 50\% nos primeiros 3-6 meses após o TxR. ${ }^{8}$ No entanto, $25 \%$ dos pacientes, após 1 ano, persistem com os níveis de PTH elevados, relacionado ao tempo de diálise e à gravidade do HPS antes do transplante (provável hiperplasia nodular). ${ }^{1,9}$ Outra causa de secreção aumentada de PTH é a disfunção do enxerto que leva ao desenvolvimento do HPS “de novo", ${ }^{10}$ estando associado à taxa de filtração glomerular (TFG) entre $40-50 \mathrm{~mL} / \mathrm{min}$, levando a alterações ósseas semelhantes às do período dialítico. ${ }^{11} \mathrm{~A}$ hipovitaminose D também colabora para o aumento da secreção do PTH, sendo sua reposição importante no controle do HPS. ${ }^{12,13}$ A persistência do HPS é a principal causa do aumento da perda de massa óssea nos pacientes transplantados estáveis, principalmente em coluna vertebral. ${ }^{14,15}$

Normalmente, observamos a evolução dos níveis séricos de Ca e PTH até o final do primeiro ano e, se persistirem elevados, há indicação de paratireoidectomia $(\mathrm{PTx}) .{ }^{16}$ Porém, em situações de hipercalcemia grave, $\mathrm{Ca}>14 \mathrm{mg} / \mathrm{dL}$ ou Cai $>1,8 \mathrm{mmol}$, e principalmente se estiver associada à perda progressiva e inexplicada da função renal, a PTx deve ser antecipada.

A recuperação da função renal contribui para melhorar e, às vezes, normalizar os níveis de calcitriol. No entanto, esse processo pode ser mais prolongado devido à terapia imunossupressora, especialmente os inibidores de calcineurina, que causam diminuição do fluxo glomerular e toxicidade tubular, e/ou os corticosteroides que diminuem a atividade da $1 \alpha$-hidroxilase e aumentam a síntese de enzimas envolvidas no catabolismo da 25-hidroxivitamina D. ${ }^{17,18} \mathrm{~A}$ incidência de hipovitaminose $\mathrm{D}$ nos pacientes transplantados está em torno de $50 \%$, e sua causa é multifatorial, devido à baixa exposição solar e ao uso frequente de bloqueador solar. Há uma associação entre os níveis de 25 -vit $\mathrm{D}$ e a função do enxerto renal, podendo a hipovitaminose $\mathrm{D}$ ser um preditor de piora de função do enxerto. ${ }^{19}$

Após o $1^{\circ}$ ano de TxR, existem poucos dados na literatura com relação às alterações dos DMO, e a frequência da monitoração deve ser semelhante àquela 
dos pacientes não transplantados com DRC, podendo ser aumentada na dependência das alterações existentes e tratamento instituído. ${ }^{20,21}$ Como mencionado anteriormente, o P está estável, o Ca tende a ser um pouco mais elevado e FA e PTH se encontram na dependência da função renal. ${ }^{24,25}$ Encontramos, no TxR tardio, hipercalcemia em $25 \%$ dos pacientes e HPS persistente em $24 \%-75 \%$ dos pacientes, de acordo com a função renal residual. ${ }^{22,23,26} \mathrm{~A}$ hipovitaminose D correlaciona-se com maiores níveis de PTH, independentemente do grau de função renal. ${ }^{22,25}$ Outra alteração importante é a acidose metabólica, associada com HPS e disfunção do enxerto. ${ }^{26}$

Os corticosteroides são uma droga deletéria ao tecido ósseo, estando associados à perda de massa óssea e inciência de fraturas. Os corticosteroides diminuem a formação óssea, promovendo menor diferenciação e função dos osteoblastos e maior apoptose de osteoblastos e osteócitos. ${ }^{27,28}$ Além disso, estimulam indiretamente a osteoclastogênese, atuando no eixo ligante do receptor ativador do NFkB (RANKL) e osteoprotegerina (OPG). ${ }^{29}$ Os corticosteroides favorecem o desenvolvimento ou manutenção do HPS por diminuírem a absorção intestinal de Ca e aumentarem a calciúria. São uma droga que também induz o hipogonadismo hipogonadotrófico, diminuindo a ação trófica dos hormônios sexuais sobre o tecido ósseo. Os efeitos dos corticosteroides no metabolismo ósseo de pacientes TxR são precoces, analisados pela densitometria óssea (DEXA) $)^{30,31}$ e biópsia óssea. ${ }^{32,33}$ Vários protocolos de imunossupressão usam doses reduzidas ou preconizam interrupção precoce dos corticosteroides após o TxR, sem alteração da função do enxerto e com diminuição da perda óssea, assim como menor incidência de necrose óssea. ${ }^{34}$

Os inibidores de calcineurina, como CSA e FK, atuam nas células ósseas através de mecanismos complexos e pouco compreendidos. Os estudos existentes avaliaram um número pequeno de pacientes, não foram desenhados para investigar os efeitos dos imunossupressores sobre o tecido ósseo e apresentam resultados contraditórios. ${ }^{35-37}$

A ação da rapamicina no tecido ósseo também é pouco conhecida. Estudo recente mostra o papel da rapamicina estimulando a proliferação de osteoblastos, entretanto; estes achados divergem com os de outros autores..$^{38,39}$

Os transplantados apresentam um risco de perda de massa óssea elevado, pois além dos mesmos fatores observados na população geral, ainda são acrescentados outros como receptores de doadores falecidos, tempo em diálise e de transplante, persistência do HPS, drogas imunossupressoras e número de mismatches ${ }^{40,41}$ As principais consequências da diminuição da massa óssea são maior risco de fraturas e incidência de doenças cardiovasculares (DCV). A incidência de fraturas entre os pacientes transplantados varia de $7 \%$ a $24 \%$, com um risco de fraturas cinco vezes maior que na população geral, sendo até 23 vezes maior quando analisada a coluna vertebral. ${ }^{42-44}$

É bem estabelecida a associação de osteoporose e risco de fraturas, detectada pela DEXA, em pacientes menopausadas, em uso de corticoides e em outros transplantados, como de coração e fígado. Entretanto, essa associação não é verdadeira para o transplantado renal, visto que a doença óssea pós-TxR é dependente da persistência dos DMO, do período dialítico e da função do enxerto. Assim, nessa população, uma densidade mineral óssea (DMO) normal não afasta o risco de fraturas, pois podem apresentar alterações na microarquitetura óssea não detectadas pela DEXA. ${ }^{28,45}$ Os resultados de estudos da DMO em transplantados tardios são variáveis, mostrando uma perda de massa óssea importante nos primeiros 6-12 meses pós-TxR, e que se mantém de forma contínua, em proporção menor, por alguns anos. ${ }^{46,47}$ A realização de DEXA está indicada em pacientes com função preservada do enxerto (DRC I-III T); no entanto, pode ser útil quando avaliada de modo seriado, em alguns pacientes em outros estágios da DRC.

Estudos realizados com biópsia óssea mostraram uma incidência de doença de alta remodelação óssea em $14 \%$ a $59 \%$ dos pacientes, associada a maior tempo em diálise, persistência do HPS e uso de inibidores de calcineurina. Por outro lado, de 10,5\% a $75 \%$ dos pacientes apresentaram doença de baixa remodelação, associada à osteoporose e ao retardo na mineralização óssea. A diminuição da DMO no TxR tardio varia de $22 \%-56 \%$, sendo maior nos idosos e nos pacientes com mais tempo em diálise e de transplante. ${ }^{45,48}$

A indicação de biópsia óssea pós-TxR deve ser feita na vigência de fraturas atraumáticas, suspeita de defeito de mineralização e na possibilidade do uso de bisfosfonatos para afastar doença óssea de baixa remodelação.

Em razão da complexidade da fisiopatologia da perda de massa óssea pós-TxR, ainda não se dispõe 
de nenhum estudo de intervenção clínica com impacto na diminuição do número de fraturas, internações ou mortalidade nesses pacientes. De maneira geral, os estudos mostram efeitos benéficos de associações de vitamina $\mathrm{D}_{2}$ ou $\mathrm{D}_{3}$, calcitriol e $\mathrm{Ca}^{49-51}$ ou do uso prolongado de calcitriol e $\mathrm{Ca},{ }^{62}$ sem resultados superiores de um ou de outro esquema terapêutico.

Os bisfosfonatos são agentes terapêuticos que inibem a função osteoclástica, promovendo um desacoplamento entre a formação e a reabsorção óssea, induzindo a uma doença óssea de baixa remodelação ou defeito de mineralização. Dessa forma, há restrição para o uso dessas drogas, pois o paciente pode apresentar uma DMO baixa sem ter uma remodelação óssea aumentada, e os bisfosfonatos poderiam levar ou agravar a baixa remodelação óssea. Além disso, são drogas de efeito acumulativo no tecido ósseo, restringindo sua indicação a pacientes com DRC I-III T. Os estudos com bisfosfonatos (pamidronato, ibandronato ou risedronato) usados na prevenção ou no tratamento da perda de massa óssea no primeiro ano do TxR apresentam nível de evidência moderado, efeito benéfico, principalmente considerando a região lombar ${ }^{52-55}$ e sem alteração na função do enxerto. Os benefícios do uso dos bisfosfonatos, nos pacientes TxR tardio, ainda são controversos: são estudos pequenos, em diferentes fases de função renal, não mostrando superioridade com relação ao tratamento com $\mathrm{Ca}+$ calcitriol. ${ }^{56}$ Não foi definido também se o risco de baixa remodelação óssea ou retardo na mineralização óssea compensariam a preservação do volume ósseo e possível redução do risco de fraturas.

\section{REFERÊNCIAS}

1. Evenepoel P, Claes K, Kuypers D, Maes B, Bammens B, Vanrenterghem Y. Natural history of parathyroid function and calcium metabolism after kidney transplantation: a single-centre study. Nephrol Dial Transplant 2004; 19:1281-7.

2. Kanaan N, Claes K, Devogelaer JP et al. Fibroblast growth factor-23 and parathyroid hormone are associated with post-transplant bone mineral density loss. Clin J Am Soc Nephrol 2010; 5(10):1887-92.

3. Evenepoel P, Meijers BKI, de Jonge $\mathrm{H}$ et al. Recovery of hyperphosphatoninism and renal phosphorus wasting one year after successful renal transplantation Clin J Am Soc Nephrol 2008; 3: 1829-36

4. Ojo AO, Hanson JA, Wolfe RA, Leichtman AB, Agoda LY, Port FK. Long-term survival in renal transplant recipients with graft function. Kidney Int 2000; 57:307-13.

5. Gwinner W, Suppa S, Mengel M et al. Early calcification of renal allografts by protocol biopsies: causes and clinical implications. Am J Transplant 2005; 5:1934-41.
6. Evenepoel P, Lerut E, Naesens M et al. Localization, etiology and impact of calcium phosphate deposits in renal allografts. Am J Transplant 2009 Nov;9(11):2470-8. Epub 2009 Aug 14.

7. Pinheiro HS, Câmara NO, Osaki KS, De Moura LA, Pacheco-Silva A. Early presence of calcium oxalate deposition in kidney graft biopsies is associated with poor long-term graft survival. Am J Transplant 2005; 5(2):323-9.

8. Bonarek H, Merville P, Bonarek M et al. Reduced parathyroid functional mass after successful kidney transplantation. Kidney Int 1999; 56:642-9.

9. Marcén R, Ponte B, Rodríguez-Mendiola $\mathrm{N}$ et al. Secondary hyperparathyroidism after kidney transplantation: a cross-sectional study. Transplant Proc 2009; 41(6):2391-3.

10. Bellorin-Font E, Rojas E, Carlini RG, Suniaga O, Weisinger JR. Bone remodeling after renal transplantation. Kidney Int Suppl 2003 Jun;(85):S125-8.

11. Akaberi S, Lindergard B, Simonsen U, Nyberg G. Impact of parathyroid hormone on bone density in long-term renal transplant patients with good graft function. Transplantation 2006; 82:749-52.

12. Lim WH, Coates PS, Russ GR, Coates PT. Hyperparathyroidism and vitamin D deficiency predispose to bone loss in renal transplant recipients. Transplantation 2009; 88(5):678-83.

13. Canalis E, Mazziotti G, Giustina A, Bilezikian JP. Glucocorticoid-induced osteoporosis: pathophysiology and terapy. Osteoporosis Int 2007; 18:319-28.

14. Bubenicek P, Sotornik I, Vitko S, Teplan V. Early bone mineral density loss after renal transplantation and pre-transplant PTH: a prospective study. Kidney Blood Press Res 2008; 31(3):196-202.

15. Torregrosa JV, Campistol JM, Montesinos M, Pons F, Martinez de Osaba MJ. Evolution of bone mineral density after renal transplantation: related factors. Nephrol Dial Transplant.1995; 10(Suppl6):111-3.

16. Triponez F, Clark OH, Vanrenthergem Y, Evenepoel P. Surgical treatment of persistent hyperparathyroidism after renal transplantation. Ann Surg 2008; 248(1):1830. Review.

17. Sato T, Fukagawa M, Uchida K et al. 1,25-dihydroxyvitamin D synthesis after renal transplantation: the role of fibroblast growth factor 23 and cyclosporine.Clin Transplant 2009; 23(3):368-74.

18. Dhawan P, Christakos S. Novel regulation of 25-hydroxyvitamin D3 24-hydroxylase $(24(\mathrm{OH})$ ase $)$ transcription by glucocorticoids: cooperative effects of the glucocorticoid receptor, C/EBP beta, and the Vitamin D receptor in 24(OH)ase transcription. J Cell Biochem 2010; 110(6):1314-23.

19. Sezer S, Yavuz D, Canoz MB, Ozdemir FN, Haberal M. Vitamin D status, bone mineral density, and inflammation in kidney transplantation patients. Transplant Proc 2009; 41(7):2823-5.

20. Kidney Disease: Improving Global Outcomes (KDIGO) CKD-MBD Work Group. KDIGO clinical practice guideline for the diagnosis, evaluation, and treatment of chronic clinical disease-mineral and bone disorder (CKD-MBD). Chapter 5: Evaluation and treatment of kidney transplant bone disease. Kidney Int 2009; 76(Supl113):S100-10.

21. Uhlig K, Berns JS, Kestenbaum B et al. KDOQI US commentary on the 2009 KDIGO clinical practice guideline for the diagnosis, evaluation, and treatment of 
CKD-mineral and bone disorder (CKD-MBD). AJKD 2010; 55:773-99.

22. Stavroulopoulos A, Cassidy MJ, Porter AJ et al. Vitamin $\mathrm{D}$ status in renal transplant recipients. Am J Transplant 2007; 7:2546-52.

23. Egbuna OI, Taylor JG, Bushinsky DA et al. Elevated calcium phosphate product after renal transplantation is a risk for graft failure. Clin Transplant 2007; 21:558-66.

24. Sprague SM, Belozeroff V, Danese MD, Martin LP, Olgaard K. Abnormal bone and mineral metabolism in Kidney transplant patients. Am J Nephrol 2008; 28:246-53.

25. Reinhardt W, Bartelworth $\mathrm{H}$, Jockenhovel $\mathrm{F}$ et al. Sequential changes of biochemical bone parameter after kidney transplantation. Nephrol Dial Transplant 1998; $13(2): 436-42$.

26. Yakupoglu HY, Corsenca A, Wahl P, Wüthrich RP, Ambühl PM. Posttransplant acdosis and associated disorders of mineral metabolism in patients with a renal graft. Transplantation 2007; 84:1151-7.

27. Canalis E. Mechanisms of glucocorticoid-induced osteoporosis. Curr Opin Rheumatol 2003; 15:454-7.

28. Cunningham J. Posttransplantation bone disease. Transplantation 2005; 79:629-34.

29. Hofbauer LC, Gori F, Riggs L et al. Stimulation os osteoprotegerin ligand and inhibition of osteoprotegerin production by glucocorticoids in human osteoblastic lineage cells: potential paracrine mechanisms of glucocorticoid-induced osteoporosis. Endocrinology 1999; 140:4382-9.

30. Horber FF, Casez JP, Steiger U, Czerniak A, Montandon A, Jaeger P. Changes in bone mass early after kidney transplantation. J Bone Miner Res 1994; 9:1-9.

31. Grotz WH, Mundinger FA, Gugel B, Exner VM, Kirste G, Schollmeyer PJ. Bone Mineral density after kidney transplantation. A cross-sectional study in 190 graft recipients up to 20 years after transplantation. Transplantation 1995; 59:982-6.

32. Rojas E, Carlini RG, Clesca P et al. The pathogenesis of osteodystrophy after renal transplantation as detected by early alterations in bone remodeling. Kidney Int 2003; 63:1915-23.

33. Velasquez-Forero F, Mondragon A, Herrero B, Pena JC. Adynamic bone lesion in renal transplant recipients with normal renal function. Nephrol Dial Transplant. 1996; 11(Suppl3):58-64.

34. Opelz G, Dohler B, Laux G, Collaborative Transplant Study. Long-term prospective study of steroid withdrawal in kidney and heart transplant recipients. Am J Transplant 2005; 5:720-8.

35. Cvetkovic M, Mann GN, Romero DF et al. The deleterius effects of long-term cyclosporine A, cyclosporine $\mathrm{G}$, and FK506 on bone mineral metabolism in vivo. Transplantation 1994; 57:1231-7.

36. Westeel FP, Mazouz H, Ezaitouni F et al. Cyclosporine bone remodeling effect prevents steroid osteopenia after kidney transplantation. Kidney Int 2000; 58:1788-96.

37. Inoue $\mathrm{T}$, Kawamura I, Matsuo $\mathrm{M}$ et al. Lesser reduction in bone mineral density by the immunosuppressant, FK506, compared with cyclosporine in rats. Transplantation 2000; 70:774-9.

38. Ogawa T, Tokuda M, Tomizawa K et al. Osteoblastic differentiation is enhanced by rapamycin in rat osteoblast-like osteosarcoma (ROS 17/2.8) cells. Biochem Biophys Res Commun 1998; 249:226-30.
39. Campistol JM, Holt DW, Epstein S, Gioud-Paquet M, Rutalt K, Burke JT. The Sirolimus European Renal Transplant Study Group. Bone metabolism in renal transplant patients treated with cyclosporine or sirolimus. Transplant Int 2005; 18:1028-35.

40. Nikkel LE, Hollenbeak CS, Fox EJ, Uemura T, Ghahramani N. Risk of fractures after renal transplantation in the United States. Transplantation 2009; 87(12):1846-51.

41. Opelz G, Döhler B. Association of mismatches for HLADR with incidence of posttransplant hip fracture in kidney transplant recipients. Transplantation 2010; .[Epub ahead of print]

42. Ramsey-Goldman R, Dunn JE, Dunlop DD et al. Incresed risk of fracture in patients receiving solid organ transplants. J Bone Miner Res 1999; 14:456-63.

43. O'Shaughnessy EA, Dahl DC, Smith CL e Kasiske BL. Risk factors for fracture in kidney transplantation. Transplantation 2002; 74:362-6.

44. Braga Junior JWR, Neves RMS, Pinheiro MM et al. Prevalence of low trauma fracture in long-term kidney transplant patients with preserved renal function. Braz J Med Biol Res 2006; 39:137-47.

45. Durieux S, Mercadal L, Orcel P et al. Bone mineral density and fracture prevalence in long-term kidney graft recipients. Transplantation 2002; 74:496-500.

46. Brandenburg VM, Ketteler M, Heussen N et al. Lumbar bone mineral density in very long-term renal transplant recipients: Impact of circulanting sex hormones. Osteoporos Int 2005; 16:1611-20.

47. Carlini RG, Rojas E, Weisinger JR et al. Bone disease in patients with long term transplantation and normal renal funtion. Am J Kidney Dis. 2000; 36(1):160-6.

48. Montalban C, Francisco ALM, Marinoso ML et al. Bone disease in long-term adult kidney transplant patients with normal renal function. Kidney Int. 2003; 63(Suppl 85):S129-32.

49. De Sevaux RG, Hoitsma AJ, Corstens FH et al. Treatment with vitamin $\mathrm{D}$ and calcium reduces bone loss after renal transplantation: a randomized study. J Am Soc Nephrol 2002; 13:1608-14.

50. Torres A, Garcia S, Gomez A et al. Treatment with intermittent calcitriol and calcium reduces bone loss after renal transplantation. Kidney Int 2004; 65:705-12.

51. Cueto-Manzano AM, Konel S, Freemont AJ et al. Effect of 1,25-dihydroxyvitamin D3 and calcium carbonate on bone loss associated with long-term renal transplantation. Am J Kidney Dis 2000; 35:227-36.

52. Walsh SB, Altmann P, Pattison J et al. Effect of pamidronate on bone loss after kidney transplantation: a randomized trial. Am J Kidney Dis 2009; 53(5):856-65.

53. Torregrossa JV, Fuster D, Monegal A et al. Efficacy of low doses of pamidronate in osteopenic patients administered in the early post-renal transplant. Osteoporos Int 2011; 22(1):281-7.

54. Grotz W, Nagel C, Poeschel D et al. Effect of ibandronate on bone loss and renal function after kidney transplantation. J Am Soc Nephrol 2001;12:1530-7.

55. Torregrossa JV, Fuster D, Gentil MA et al. Open-label trial: effect of weekly risedronate immediately after transplantation in kidney recipients. Transplantation 2010; 89(12):1476-81.

56. Jeffery JR, Leslie WD, Karpinski ME et al. Prevalence and treatment of decreased bone density in renal transplant recipients: a randomized prospective trial of calcitriol versus alendronate. Transplantation 2003; 76:1498-502. 